\title{
Typological Accounts for Nominal Forms
}

\author{
Eun-Joo Kwak \\ Sejong University
}

\begin{abstract}
Various linguistic phenomena may serve a basis for the classification of languages and nominal forms make part of the criteria for the classification. In this study, two major approaches are closely compared for language typology based on nominal forms. Chierchia (1998a, b) assumes that morpho-syntactic features should be crucial in determining the denotations and grammatical categories of NPs. He suggests three classificatory categories with the notion of nominal mapping parameters. Contrastingly, the OT analysis of de Swart \& Zwarts (2009, 2010) do not assume that morpho-syntactic features should be collapsed with countability and plurality. They propose several general constraints governing nominal forms and argue that different ordering of the constraints in optimality accounts for different nominal forms crosslinguistically. I have shown that the OT
\end{abstract}

\footnotetext{
Eun-Joo Kwak

Department of English Language and Literature, Sejong University

98 Gunja-dong, Gwangjin-gu, Seoul 143-747, Korea

Phone: +82-2-3408-3633; Email: ejkwak@sejong.ac.kr
}

Received September 8, 2011; Revised October 30, 2011; Accepted November 17, 2011. 
analysis provides an appropriate framework to categorize languages systematically.

Keywords: countability, plurality, optimality, markedness constraint, faithfulness constraint

\section{Introduction}

Cross-linguistic observations show that languages adopt different strategies to mark the grammatical notions of countability and plurality. Specific plural morphemes may be postulated, definite and indefinite articles may give a clue, or no explicit marking is allowed. In spite of the apparent diverse patterns to mark countability and plurality, languages may be classified into a few groups depending on their morphological and syntactic features.

Given the possibility of typological classification, a deeply rooted problem is how much morphology and grammar interact each other or whether explicit morphological features determine grammatical features. Two major approaches are made to deal with this problem. One is based on the view that morpho-syntactic features determine a grammar, which is represented by Chierchia (1998a, b). Languages are classified into three categories depending on argument and predicate properties of noun phrases (henceforth NPs). The other approach made by de Swart \& Zwarts $(2009,2010)$ accepts the view that morpho-syntactic features may not be crucial in determining a grammar, which is exemplified by an optimality-theoretic approach. Although the same set of constraints are postulated for languages, the ordering for their application may not be identical and thus languages show different morpho-syntactic patterns to mark grammatical features.

In this study, I will critically review problems with the typological approach of Chierchia (1998a, b) and show that the 
optimality-based account provides a better classificatory tool for language typology. I will also argue that apparent idiosyncratic patterns for countability and plurality are attributed to unusual ranking of constraints and the development of local constraints.

\section{A Morpho-Syntactic Approach to Language Typology}

\subsection{Chierchia (1998a, b)}

One of the general assumptions in semantics has been that the countability of nouns is highly dependent on the physical nature of their references. Count nouns denote discrete objects that are countable in reality while mass nouns denote dense entities such as liquids and materials that are hard to be counted in the real world. Because only discrete objects may be grouped to make plural-numbered entities, plurality is a relevant notion to count nouns but not to mass nouns. According to this assumption, three different concepts are parallel without allowing a discrepancy: the discreteness of the real world and the countability and the plurality of nouns.

One of the challenges to this simple assumption has been made by Chierchia (1998a, b). The starting point of his analysis is division between the countability of a noun and the discreteness of its reference. He argues that entities with equal perceptual salience may belong to different nominal categories. For instance, although the object denoted by rice and lentil are not distinguished in their perceptual salience, rice is mass and lentil is count. Moreover, the same objects may be lexicalized into more than one expression which has different countability. This is exemplified by pairs of a count and a mass, e.g., shoe/footwear, coin/change, and carpet/carpeting. 
Chierchia proposes that the solely grammatical notion of countability should be categorized by the 'nominal mapping parameters' of nouns: [arg] for arguments and [pred] for predicates. Since noun phrases in languages with the parameter value [+arg] denote names of kinds typed $\langle e\rangle$, bare NPs without determiners or number inflection may appear in argument positions. NPs in languages with [+pred] denote predicates of type $<e, t>$, so bare NPs do not occur in argument positions due to the type-mismatch problem. Given the parameters, languages are classified into three different categories as follows:

(1) a. $[+$ arg, +pred]: English, German

b. [-arg, +pred]: French, Italian

c. [+arg, -pred]: Chinese, Thai

In English and German, which are classified to have [+arg, + pred], both bare NPs and NPs with a determiner may occur in argument positions. French and Italian, assigned [-arg, +pred], do not allow bare NPs in argument positions while Chinese and Thai have bare NPs in argument positions due to their features [+arg, -pred].

Chierchia's language classification hinges on the view that morpho-syntactic properties determine the grammatical notions of countability and plurality. To substantiate this dependency, he enumerates properties of mass nouns. First, mass nouns do not have plural morphology. 1 Despite the similarity of their denotations, the count noun shoe has the plural form shoes but its mass

1 Chierchia (1998a, b) provides a semantic account for the lack of plural morphology for mass nouns. He assumes that mass nouns be lexically plural, denoting kinds. Because mass nouns are already plural, there is no need to add a plural morpheme to mass nouns. Part of the evidence for the plurality of mass nouns comes from the fact that the denotation of a mass collection like furniture is not distinct from a plural NP like pieces of furniture. A problem with the plurality of mass nouns has been pointed out by Kwak (2009). 
counterpart footwear is not pluralized. Second, mass nouns may not combine with numerals directly. Although the count noun drop occurs with the numeral directly as in three drops, the mass noun blood may not occur with a numeral directly like *three bloods. Finally, mass nouns need a classifier or a measure phrase when counted. For example, the mass noun rice may be counted in forms like three grains of rice or three kilos of rice. Based on these properties, Chierchia concludes that languages with [+arg, -pred], e.g., Chinese, do not have count nouns because NPs are always accompanied by additional phrases to deliver counting readings and plural forms of NPs do not occur.

\subsection{Problems with Morpho-Syntactic Based Typology}

The seminal work of Chierchia (1998a, b) provides insightful arguments on the relations between morpho-syntactic properties and the grammatical notions of countability and plurality. However, the three general categories defined by Chierchia need a further refinement to reflect the semantic properties of nouns and diverse language variations.

According to Chierchia's classification, Chinese, having [+arg, -pred], includes only mass nouns in its lexicon, which is supported by no plural morphology and the mandatory use of a classifier for counting. However, a number of counterarguments have been made against the lack of count nouns. First, classifiers may be divided into count and mass by their semantic properties ${ }^{2}$ (cf. Cheng \& Sybesma 1999, Chien et al. 2003). Count classifiers provide information on how entities are partitioned in a natural

2 According to Zhang (2007), numeral classifiers in Chinese have functions more than counting. They deliver categorization parameters such as humanness, animacy, shape, function, consistency, and size. Hence, research on classifiers should be rooted in the cognitive process of human beings (cf. Friedrich 1970, Adams \& Conklin 1973, Allen 1977). 
way, and mass classifiers are used to denote a unit of measure that quantifies potions of entities. Hence, count classifiers denote inherent or permanent properties of objects while mass classifiers denote temporary states of entities. Given the different semantic functions of count and mass classifiers, a noun may occur with more than one classifier. For instance, xiangyan 'cigarette' may occur with the count classifier gen to denote the number of cigarettes or the mass classifier bao 'pack' for the temporary grouping of cigarettes. Second, the different semantic features of count and mass classifiers are reflected in their syntax (cf. Cheng \& Sybesma 1999). The modification marker de may be inserted between a mass classifier and a noun but it is not allowed to occur with a count classifier. ${ }^{3}$
(2) a. san
bang
(de) rou
three
$\mathrm{Cl}$ pound
(De) meat
'three pounds of meat'
b. ba
tou
$(*$ de) niu
eight Cl_head
'eight cows'
(*De) cow

Rou 'meat' is a mass noun to denote a dense entity of material, and niu 'cow' is a count noun with a discrete denotation of cow. Bang for rou is a mass classifier which denotes the temporary states of meat while tou for niu is a count classifier that concerns the permanent property of the individuality of cow. This difference in countability is reflected in the optional occurrence of de. It may occur with bang but not with tou. Based on the semantic and syntactic properties of classifiers, Cheng \& Sybesma (1999) argue

3 Here is a list of abbreviations used in this article.

$\mathrm{NP}$-noun phrase; DP-determiner phrase; NumP-numeral phrase; Pl-plural; $\mathrm{Cl}$ classifier; Poss-possessive; ASP-aspect 
that while countability distinction is marked at the level of noun in Indo-European languages, it is reflected at the level of classifier in Chinese.

Another problematic language to Chierchia's classification is Dëne Suliné (henceforth Dëne), a Northern Athapaskan language spoken in Northern Canada. Wilhelm (2008) makes an observation that nouns in Dëne occur in argument positions in their bare forms without a determiner or plural morphology. Then, Dëne should be classified as a language with [+arg, -pred] like Chinese, and all nouns in Dëne are expected to be mass nouns. However, the mandatory use of a classifier or measure phrase is not part of the grammar of Dëne.

(3)
a. solághe
ejëretth'úé t1l
/ *solághe
ejëretth'úé
five
milk
container/ five
milk
'five pounds of milk/*five milk'
b. solághe k’ásba
five chicken
'five chickens'

Some nouns like ejëretth 'milk,' a counting reading is not available without the occurrence of a measure phrase like tll 'container.' However, nouns like k'ásba 'chicken' combine with a numeral directly. Moreover, nouns that need a measure phrase for counting denote liquids, substances, or abstract concepts while nouns which do not need a measure phrase make a group which are similar to that of count nouns in English. Hence, Whihelm argues that nouns in Dëne should be divided into count and mass nouns in spite of the lack of plural morphology and the occurrences of bare NPs in argument positions.

Korean and Japanese pose a more problem to Chierchia's classification in that the use of a plural morpheme and a classifier is optional. In Chierchia's analysis, the nominal mapping parameters determine the classificatory category of a language, 
which does not leave room for the optionality of plural forms or classifier occurrences. If count nouns in plural readings may or may not occur with a plural morpheme in a given language, the mapping parameters for the language are not set to specific values. Then, the language does not belong to any of the categories, which reveals the flexibility problem of Chierchia's classification.

The three different patterns of the problematic cases to Chierchia's analysis show that the grammatical notions of countability and plurality do not hinge on the morpho-syntactic properties of NPs. An approach based on a different view is required for the typological argument of languages.

\section{An Optimality-Based Approach to Language Typology}

\subsection{Optimality Theory Semantics}

For the analysis of phonological data, 'Optimality Theory' (henceforth OT) has been proposed by Prince \& Smolensky (1993). Later, it turns out that the principle of OT is applicable to other areas such as syntax, semantics, and pragmatics. According to the notion of optimality, speakers are supposed to select the optimal form for a given meaning and hearers are supposed to take the optimal interpretation for a given form. Since the optimization on the side of speakers may not coincide with that of hearers, constraints in the OT framework may be in conflict. To resolve this conflict, constraints are assumed soft and violable in the OT analysis. Low-ranked constraints are ready to be violated when they are in conflict with more important or highly ranked ones.

To capture the universality of languages, the same set of constraints which are hierarchically ordered is posited for 
languages. However, relative ordering among the constraints are not inherently determined. Hence, languages show diversity in the ranking and allow different nominal forms depending on which constraint is highly ordered. To see how universality and diversity are balanced in languages, let us consider what kind of constraint is necessary.

Depending on languages, bare forms are preferred over NPs marked for grammatical information such as plurality and definiteness. To account for this preference, de Swart \& Zwarts $(2009,2010)$ propose the general markedness constraint *FunctN.

(4) *FunctN: Avoid functional structure in the nominal domain.

Since *FunctN bars the grammatical specification of NPs, a nominal structure which does not include any functional category is judged to be the most optimal. Suppose that there are four syntactic structures for NPs.

(5) a. $[\mathrm{NP} N]$

b. [NumP Num [NP N]]

c. [DP D [NP N]]

d. [DP D [NumP Num [NP N]]]

(5a) is the simplest structure without including any functional category, so it does not violate *FunctN at all. (5b) and $(5 c)$ contain one functional category while (5d) occurs with two functional categories of the determiner phrase (DP) and the number phrase (NumP). Thus, (5d) is judged to be the least preferred form according to * FunctN.

Along with the general markedness constraint, several faithfulness constraints are needed to account for optimal forms. First, nominal forms may be distinct by plurality, which is captured by FPL (cf. de Swart \& Zwarts 2009, 2010). 
(6) FPL: Reference to a group of individuals must be reflected in a special plural form of the nominal.

FPL states that NPs should be morphological distinguished by their plurality and special plural morphology is mandatory. Note that explicit morphology is not required for singulars. ${ }^{4}$ Here, plural NPs are regarded as marked forms.

Second, definiteness also serves as a criterion to make different nominal forms (cf. de Swart \& Zwarts 2009, 2010).

(7) FDEF: Reference to discourse unique individuals (unique/ maximal or familiar ones) requires the use of an expression of definiteness.

De Swart \& Zwarts argue that a definite article plays many roles including uniqueness, maximality, or familiarity. ${ }^{5}$ Hence, they propose the term 'discourse uniqueness,' on which the faithfulness constraint FDEF is based. Following FDEF, NPs take different forms by definiteness, and explicit marking for definite NPs is compulsory. However, the morphological specification for indefiniteness is not mandatory according to FDEF.

Finally, counting readings may be delivered only by the occurrence of a numeral in a nominal structure. However, it may be preceded or followed by a classifier or measure phrase to make counting more explicit. Hence, another faithfulness constraint is

4 Typological study including Greenberg (1966) and Corbett (2000) shows that if there is only one marked form for the pair of a singular and a plural, it is always the plural that takes the marked form. FPL is proposed based on this typological observation.

5 Uniqueness by a definite article is exemplified by a phrase like the queen of the Netherlands, which refers to the only queen in the Netherlands. On the other hand, the definiteness of the stars is used to denote a maximal group consisting of all the stars. The dog delivers familiarity, occurring in a discourse like I saw a dog in the park, and the dog wagged its tail. 
proposed (cf. Kwak To Appear).

(8) FCL: The occurrence of a numeral is accompanied by a classifier or measure phrase.

FCL states that the counting reading of a numeral is more specified by the occurrence of a classifier or measure phrase. Depending on languages, FCL applies to all the nouns or it is relevant to a group of nouns, e.g., mass nouns. When the application of FCL is varied by the grammatical or semantic features of nouns, the constraint may be further divided into a few local constraints which are ordered differently in their optimality.

\subsection{Nominal Forms in English and Chinese}

Crosslinguistic studies show that languages have diverse patterns of nominal forms in their grammar while sharing some common aspects. In the morpho-syntactic approach of Chierchia (1998a, b), the common aspect is instantiated by the nominal mapping parameters and language variations are reflected in the values of the parameters. In the OT analysis, the universal aspect of languages is captured by the same set of constraints while language variations are attributed to the different ranking of the constraints.

To get the better picture of the OT analysis proposed by de Swart \& Zwarts (2009, 2010), let us compare different plural forms between English and Chinese.

(9) a. I saw a bear/bears.

b. Wò kàngjiàn xióng le I see bear ASP

'I see a bear/some bears/the bear(s).'

In English, singular and plural NPs are explicitly distinguished 
by the occurrence of the plural morpheme ' $-s$ ' and that of the indefinite article. 6 However, since plurality is not overtly marked in Chinese, the bare NP xióng 'bear' is interpreted as either singular or plural. As discussed in the previous section, the optimal forms of NPs are determined by the interaction of the markedness constraint *FunctN and the faithfulness constraint FPL, which are in conflict. Hence, depending on their relative ranking, one of them is decisive in determining optimal nominal forms while the other is violated. The marked form of the plural NP in (9a) shows that FPL is considered more important than *FunctN in English. On the other hand, the unmarked form in (9b) shows that *FunctN has a priority over FPL in determining nominal forms.

(10) a. FPL $>*$ FunctN

(English)

b. *FunctN $>$ FPL

(Chinese)

FPL outranks *FunctN in English while *FunctN takes a higher position than FPL in Chinese.

Given the ranking of the constraints, the procedure of deriving an optimal plural form in English is represented as follows:

Table 1. Plural Marking in English

\begin{tabular}{|c|c|c|c|}
\hline$\exists \mathrm{x} \_\mathrm{pl}[\operatorname{bear}(\mathrm{x}) \wedge \operatorname{see}(\mathrm{x})(\mathrm{I})]$ & Form & FPL & *FunctN \\
\hline & I saw bear & $*$ & \\
\hline & I saw bears & & $*$ \\
\hline
\end{tabular}

6 De Swart \& Zwarts $(2009,2010)$ note that some category of nouns does not show distinction as to plurality. Nouns for animals that are fished on or hunted, e.g., carp and salmon, take bare forms even in plural readings. Hence they posit a local markedness constraint ${ }^{*} \mathrm{PL}_{\mathrm{FISH}}$ to block the occurrence of the plural morpheme for this category of nouns and locate it higher than FPL in the ranking. In the OT analysis, local constraints are useful to deal with idiosyncratic behaviors shown in languages. 
The first column of the table shows the intended meaning, which amounts to say 'I saw bears.' The second column includes two possible forms to deliver this meaning, one with the bare NP bear and the other with the plural NP bears. Each of the forms violates one of the constraints. The bare NP is not marked for plurality and thus violates FPL, which is marked by the asterisk. The plural NP does not follow *FunctN and gets an asterisk in the fourth column. Although the number of constraints the two NPs violate is equal, the bare NP violates the highly ranked constraint. Hence, the plural form is judged optimal in English, which is shown by the pointing hand ( $\vec{s}$ ).

To deliver the same meaning, two nominal forms may be considered in Chinese as given in the second column of Table 2.

Table 2. Plural Marking in Chinese

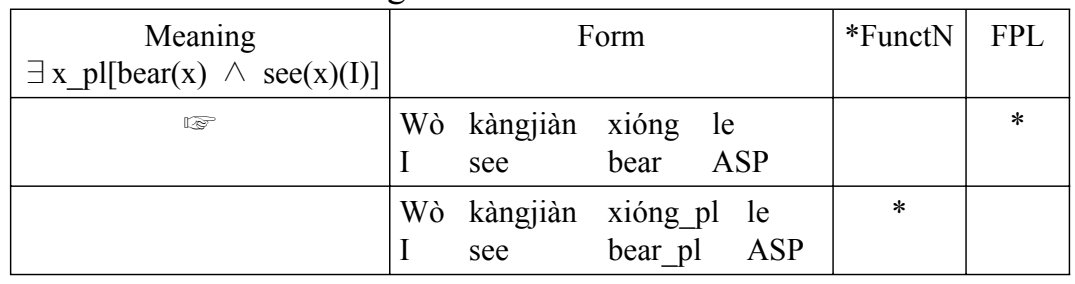

The bare NP in the second row violates *FPL while the plural form in the third row does not meet *FunctN. Although the constraints they violate are identical to those of English, their ordering is reversed in Table 2. The violation of FPL is more tolerable in Chinese. Thus, the bare NP is regarded as optimal.

Nominal forms in English are distinguished not only by plurality but also by definiteness. Definite NPs are always preceded by the definite article while indefinite NPs are preceded by the indefinite article or a zero determiner. Unlike English, Chinese NPs are not marked for definiteness. For example, the bare NP xióng in (9b) is construed as either an indefinite NP 'a bear/bears' or a definite 
one 'the bear(s).' The distinct nominal patterns between English and Chinese are again reflected in the different ordering of constraints. FDEF, which opts for NPs marked for definiteness, is ranked higher than ${ }^{*}$ FunctN in English whereas it takes a lower position than *FunctN in Chinese.

(11) a. $\{$ FPL, FDEF $\}>>$ *FunctN (English)

b. ${ }^{*}$ FunctN $>$ FPL, FDEF $\}$ (Chinese)

The relative ordering between FPL and FDEF is not meaningful in both of the languages, which is represented by the bracket. Definite NPs in bare forms violate the higher constraint FDEF in English, and thus they are not regarded as optimal. However, bare NPs in definite readings satisfy the higher constraint ${ }^{*}$ FunctN in Chinese, so they are optimal forms.

Finally, the occurrence of a classifier is restricted to mass nouns in English. Hence, I argue that FCL is divided into two local constraints: FCL MASS and FCL COUNT. The mandatory use of a classifier for mass nouns means that FCL MASS outranks its conflicting constraint *FunctN. The lack of a classifier for count nouns means that $\mathrm{FCL}_{\text {COUNT }}$ is lower ranked than *FunctN. Here is the ordering of the constraints for English.

(12) a. $\{$ FPL, FDEF, FCL MAss $\}>*_{\text {FunctN }}>$ FCL $_{\text {COUnT }}$ (English)

b. FCL $>*$ FunctN $\gg\{$ FPL, FDEF $\}$ (Chinese)

Unlike English, all the nouns in Chinese occur with a classifier, so the general constraint FCL is operative in Chinese. Furthermore, FCL should outrank *FunctN to make the use of a classifier obligatory. 


\subsection{Nominal Forms in Dëne and Korean}

As pointed out in section 2.2., one of the problematic languages to Chierchia's analysis is Dëne, in which no number marking is allowed but the use of a classifier is different depending on the countability of nouns. Here are the data repeated from section 2.2.
a. solághe k'ásba
five chicken
'five chickens'
b. solághe ejëretth'úé t1l1/ *solághe ejëretth'úé five milk container/ five milk 'five pounds of milk/*five milk'

The denotation of k'ásba is a set of chickens, which are discrete in the real world. Although $k^{\prime} a ́ s b a$ is not followed by any plural morpheme, it combines with the numeral without the occurrence of a classifier. On the other hand, ejëretth 'úe refers to milk, which is dense and hard to be counted by itself. When counted, it requires the occurrence of a classifier or measure phrase like t1l. Direct counting only with a numeral is not allowed to this noun.

In the OT analysis, countability does not hinge on plural morphology. Suppose that nouns in Dëne are divided into count and mass nouns, following Wilhelm (2008). Then, I argue that the lack of plural morphology is attributed to the lower ranking of FPL than *FunctN. Count nouns may be plural but their plurality is not morphologically marked due to the lower ranking of FPL. Moreover, since classifiers are mandatory only for mass nouns in counting readings, FCL is divided into two local constraints: FCL $L_{\text {MASS }}$ and $F C L_{\text {COUNT. }}$ To render a classifier to follow a mass noun, $\mathrm{FCL}_{\mathrm{MASS}}$ is ranked higher than *FunctN, which again outranks FCL count nouns. As a result, the relevant ordering for the constraints should be like the following: 


\section{(14) $\mathrm{FCL}_{\mathrm{MASS}} \gg *$ FunctN $\gg\left\{\right.$ FPL, FCL $\left.\mathrm{FCOUNT}_{\text {T }}\right\}$ (Dëne)}

The last category of languages that poses a problem to Chierchia's framework includes Korean, in which a plural morpheme and classifiers are optionally used. This optionality makes it very awkward to determine the values of the nominal mapping parameters and make a typological classification of the language. Note that the explicit occurrence of the plural morpheme -tul makes it clear that the noun is plural. However, a bare noun without being followed by -tul is ambiguous between a singular and a plural.

\section{(15) haksayng/ haksayng-tul student/ student-P1 'a student or (the) students/(the) students'}

The ambiguity of haksayng contrasts with the plural reading of haksayngtul in (15). To accommodate the optional use of the plural morpheme, Kwak (2011) proposes that FPL need to be coranked with *FunctN.

(16) $\{*$ FunctN, FPL $\}$ (Korean)

When *FunctN and FPL are not distinguished in their ranking, the violation of the constraints is regarded as having equal optimality. The plural NP haksayngtul violates *FunctN while the bare NP haksayng does not satisfy FPL. Both of the NPs violate one constraint and the level of the hierarchical ranking of the constraints is not distinct. Hence, the two NPs are judged to have the same degree of optimality and used alternately.

As for definiteness, Korean has the definite article $k u$ in the grammar to mark definite NPs explicitly. However, definite NPs in Korean are not always preceded by this article. The bare NPs in (15) are ambiguous between definite or indefinite readings. 
Then, FDEF should also be coranked with its conflicting constraint *FunctN to make the occurrence of $k u$ optional. Then, the ordering of the constraints is elaborated as follows:

\section{(17) $\{*$ FunctN, FPL, FDEF $\}$ (Korean)}

Interestingly, the optionality of the plural morpheme does not hold in some cases. When an NP is definite, its plural reading is conveyed only through the occurrence of -tul. Compare the ambiguity of haksayng in (15) and the rigid interpretations of the NPs in (18) (cf. Song 1975, Nemoto 2005, Kwak 2010).

\section{(18) $\mathrm{ku}$ haksayng/ ku haksayng-tul the student/ the student-Pl 'the student/the students'}

$K u$ haksayng does not have a plural reading. The rigidity shown in (18) suggests that FPL in Korean needs to be subcategorized by definiteness, i.e., FPLDEF and FPL INDEF. Moreover, FPL be ranked over *FunctN to make the occurrence of -tul mandatory for definite NPs.

\section{(19) $\mathrm{FPL}_{\mathrm{DEF}}>>\left\{{ }^{*}\right.$ FunctN, FDEF, FPL $\left.\mathrm{L}_{\mathrm{INDEF}}\right\}$ (Korean)}

The ambiguity of indefinite NPs as to plurality is captured by the coranking of FPL INDEF and *FunctN.

Finally, the use of a classifier is available for all nouns regardless of their grammatical or semantic features in Korean. However, it is obligatory for mass nouns.

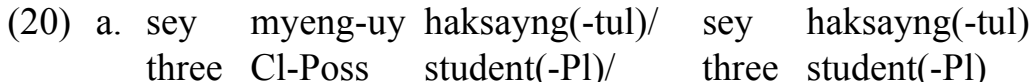
'three students' 
b. sey byeng-uy mwul/ *sey mwul three Cl-Poss water/ three water 'three bottles of water/*three water'

The count noun of haksayng may or may not occur with the classifier, but the lack of the classifier makes the mass noun $m w u l$ awkward in a counting reading. Thus, FCL is also divided into two local constraints by countability, FCL COUNT $_{\text {namely and }}$

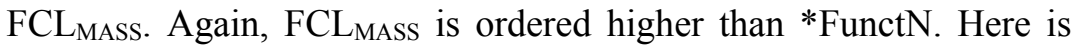
the final ranking of the constraints for Korean.

(21) $\left\{\right.$ FPL $\left._{\text {DEF }}, \mathrm{FCL}_{\mathrm{MASS}}\right\}>>$ *FunctN, FDEF, FPL INDEF, FCL $\left.L_{\text {COUNT }}\right\}$ (Korean)

Dual nominal forms allowed in Korean, which are hard to be accounted for in Chierchia's analysis, are attributed to the coranking of the markedness and the faithfulness constraints. The coranking implicates that the violation of the either of the constraints has the equal degree of optimality and does not bar the occurrence of violated nominal forms in Korean. Furthermore, distinct plural forms by definiteness are systematically explained by the postulation of the local constraints and their distinct ordering in optimality.

\subsection{Comparisons between the Two Approaches}

The typological classification of languages is conducive to elucidating patterns of linguistic variations and the universality of languages. Among the criteria for the classification are nominal forms which may vary by number morphology and the occurrences of articles and classifiers. One analysis for language classification by nominal forms is provided by Chierchia (1998a, b). The underlying assumption in this analysis is that morphosyntactic features are reflected in the grammar of a language. To 
put it in another way, if morpho-syntactic patterns of nominal forms are different, the corresponding grammars are also distinct. A plural morpheme or a syntactic device to mark plurality is regarded as an explicit sign for the countability of nouns. Hence, Chierchia argues that languages may consist of only mass nouns when they are lack of morpho-syntactic features for count nouns. With the notion of the nominal mapping parameters, Chierchia makes three categories for language typology.

Chierchia's analysis is a monumental work in that typological classification is based on the semantic properties of expressions and extended by their morpho-syntactic features. However, his emphasis on morpho-syntactic features makes his framework vulnerable to cope with linguistic diversity and accommodate flexibility needed by idiosyncratic behaviors shown by some languages. First, Chierchia suggests common properties shared by mass nouns, according to which no plural morphology and the mandatory use of a classifier cannot be detached. However, these two properties need to be treated separately in some languages. This shows that plurality is a separate issue from countability, and furthermore morpho-syntactic features may not determine countability and plurality. Second, Chierchia's classification is too general to encompass all the languages. Diverse patterns of nominal forms shown by crosslinguistic data require more specified categories to handle them.

In contrast with Chierchia's analysis, the OT analysis of de Swart \& Zwarts $(2009,2010)$ does not assume that grammatical notions of countability and plurality should coincide with typical morpho-syntactic features for them. Languages are assumed to share the same set of constraints, which reflects the generality of languages. However, the constraints may be set to different positions in the hierarchical ordering of optimality, and morpho-syntactic features triggered by lower-ranked constraints are not specified explicitly. Different specification of the features accounts for the diversity of languages. For instance, de Swart \& 
Zwarts $(2009,2010)$ provide three categories depending on the relative ordering of ${ }^{*}$ FunctN, FPL, and FDEF.

(22) a. *FunctN $>$ FPL, FDEF $\}$ : no number morphology, no articles (Chinese)

b. $\{$ FPL, FDEF $\}>*$ FunctN: number morphology, articles (English, German, French, Hebrew, Bulgarian)

c. FPL $>$ *FunctN $>$ FDEF: number morphology, no articles (Hindi, Polish)

When the markedness constraint *FunctN outranks the other constraints, a language does not include any morpho-syntactic sign for countability and plurality and allows only bare NPs. This is exemplified by Chinese. On the other hand, the higher ranking of the faithfulness constraints FPL and FDEF includes number morphology and articles for countability or definiteness. Thus, bare NPs without any sign for countability, plurality, and definiteness are not allowed in this category of languages. As shown in (22c), the two faithfulness constraints may be separately ranked.

Along with the different ranking of the constraints, the OT analysis is equipped with another device to deal with linguistic diversity, namely the postulation of local constraints. A general constraint like FPL or FCL may be subcategorized by grammatical or semantic features. As discussed in section 3.2. and 3.3., FCL may be divided into FCL COUNT $_{\text {and }}$ FCL $_{\text {MASs, }}$ and FPL may also be divided by definiteness to deal with Korean nominal forms. The postulation of local constraints has the effect that the apparent idiosyncratic behaviors of some languages are part of the general patterns shown by other languages. This makes it possible to capture the universality and diversity of languages in a systematic way. Finally, various typological comparisons are possible depending on which constraints are selected. Not all of the constraints have to be addressed to categorize languages, but partial comparisons are also available depending on purposes. 
Therefore, different levels of comparisons are accessible in the OT analysis.

\section{Conclusions}

The typological classification of languages contributes to elucidate the universality and diversity of languages. Various linguistic phenomena serve a basis for categorizing languages and different purposes are achieved depending on which phenomena are selected for comparison. Nominal forms constitute part of the criteria to classify languages.

Two different positions may be considered for language classification by nominal forms. One is suggested by Chierchia (1998a, b), in which morpho-syntactic features are crucial to determine the denotations of NPs and the category of languages. The other is the OT analysis by de Swart \& Zwarts (2009, 2010), where the grammatical notions of countability and plurality are treated separately from the morpho-syntactic features of nominal forms. Each of the approaches has its own theoretical merits and is conducive to clarifying linguistic systems underlying crosslinguistic data.

In this study, I have made close comparisons between the two approaches and argued that the underlying assumption of Chierchia's analysis is not convincing given the counterarguments raised by other studies. Furthermore, his categorization is too general to deal with the required diversity and too rigid to encompass apparent idiosyncratic behaviors of some languages. In the OT analysis, the universality of languages is well captured by postulating the same set of constraints. It still leaves room for flexibility, assuming different hierarchical ordering of the constraints and the postulation of local constraints. I have shown that the problematic phenomena in Chierchia's analysis are well explained in the OT analysis. 


\section{References}

Adams, K. \& N. Conklin. 1973. Toward a Theory of Natural Classification. Proceedings of the $9^{\text {th }}$ Regional Meeting, Chicago Linguistic Society 1-10. Chicago, IL: Chicago Linguistic Society. Allen, K. 1977. Classifier. Language 53, 285-311.

Cheng, L. \& R. Sybesma. 1999. Bare and Not-So-Bare Nouns and the Structure of NP. Linguistic Inquiry 30, 509-542.

Chien, Y. et al. 2003. Chinese Children Comprehension of Count Classifiers and Mass Classifiers. Journal of East Asian Linguistics 12, 91-120.

Chierchia, G. 1998a. Plurality of Mass Nouns and the Notion of Semantic Parameter. In S. Rothstein (ed.), Events and Grammar 53-103. Dordrecht: Kluwer.

1998b. Reference to Kinds across Languages. Natural Language and Semantics 6, 339-405.

Corbett, G. 2000. Number. Cambridge: Cambridge University Press. Friedrich, P. 1970. Shape in Grammar. Language 46, 370-407.

Greenberg, J. 1966. Language Universals with Special Reference to Feature Hierarchies. The Hague: Mouton.

Kwak, E. 2009. A Parallel between Countability and Atomicity. The Journal of Language and Translation (Now Called The Journal of Universal Language) 10, 113-137.

. 2010. The Distributivity of Collection Terms in Korean. The Journal of Language and Translation (Now Called The Journal of Universal Language) 11, 179-205.

. To Appear. Optimal Forms of Noun Phrases in Korean. The Linguistic Association of Korea Journal 20.1.

Nemoto, N. 2005. On Mass Denotations of Bare Nouns in Japanese and Korean. Linguistics 43, 383-413.

Prince, A. \& P. Smolensky. 1993. Optimality from Neural Networks to Universal Grammar. Science 275, 1604-1610.

Song, S. 1975. Rare Plural Marking and Ubiquitous Plural Marker 
in Korean. Language Research 11, 77-86.

de Swart, H. \& J. Zwarts. 2009. Less Form More Meaning: Why Bare Nominals Are Special. Lingua 119.2, 280-295.

. 2010. Optimization Principles in the Typology of Number and Articles. In B. Heine \& H. Narrog (eds.), Handbook of Linguistic Analysis 555-582. Oxford: Oxford University Press. Wilhelm, A. 2008. Bare Nouns and Number in Dëne Suliné. Natural Language Semantics 16, 39-68.

Zhang, H. 2007. Numeral Classifiers in Mandarin Chinese. Journal of East Asian Linguistics 16, 43-59. 